\title{
SPIN-GALVANIC EFFECT AND SPIN ORIENTATION BY CURRENT IN NON-MAGNETIC SEMICONDUCTORS
}

\author{
S.D. GANICHEV \\ University of Regensburg, Regensburg, 93040 Germany \\ sergey.ganichev@physik.uni-regensburg.de \\ Received Day Month Year \\ Revised Day Month Year
}

The spin-galvanic effect and the inverse effect, which yeilds current induced spin polarization, in low dimensional semiconductor structures are reviewed. Both effect are caused by asymmetric spin relaxation in systems with lifted spin degeneracy due to $\boldsymbol{k}$-linear terms in the Hamiltonian.

Keywords: spin-galvanic effect; spin polarization; spin splitting; terahertz.

Lately, there is much interest in the use of the spins of carriers in semiconductor quantum well (QW) structures together with their charge to realize novel concepts like spintronics. Spin-orbit coupling provides a versatile tool to generate and to manipulate the spin degree of freedom in low-dimensional semiconductor structures. The spin-galvanic effect, where a nonequilibrium spin polarization causes an electric current, or the inverse process, in which an electrical current generates a spin polarization, and the spin Hall effect, where an electrical current drives a transverse spin current and causes a spin accumulation observed near the sample boundary, are all consequences of spin-orbit coupling.

The necessary conditions to develop spintronic devices are high spin polarizations in QWs and a large spin-splitting of subbands in $\boldsymbol{k}$-space, where $\boldsymbol{k}$ is electron wavevector. The latter is important for the ability to control spins with an external electric field by the Rashba effect. ${ }^{1}$ Significant progress has been achieved recently in generating high spin polarizations, in demonstrating the Rashba splitting and also in using the splitting for manipulating the spins. ${ }^{2}$ At the same time as these conditions are fulfilled it has been shown that the spin polarization itself drives a current, resulting in the spin-galvanic effect observed in gyrotropic ${ }^{\text {a }}$ low-dimensional structures. This effect, predicted by Ivchenko et al. ${ }^{3}$, was observed by Ganichev et al. applying terahertz radiation and named the spin-galvanic effect. ${ }^{4}$ The spin-galvanic effect is caused by asymmetric spin relaxation process. While spin-galvanic effect does not necessarily requires optical excitation, in experiments on spin-galvanic

aThe gyrotropic point group symmetry makes no difference between components of axial and polar vectors. 
effect nonequilibrium spin was generated by optical means applying circularly polarized radiation. ${ }^{5-7}$ The absorption of circularly polarized light results in optical spin orientation due to the transfer of the angular momentum of photons to electrons of a two-dimensional electron gas (2DEG). In this case the spin-galvanic effect represents a spin photocurrent generated by homogeneous monochromatic optical excitation.

Spin photocurrents generated by excitation with circularly polarized radiation in QWs, which occur in unbiased samples, have attracted a growing attention in the recent decade. Several mechanisms of electric currents driven by optically generated spin polarization, like circular photogalvanic effect, spin photocurrents due to quantum interference of one- and two-photon excitations and magneto-gyrotropic effect, are observed in zinc-blende-structure based quantum well structures and SiGe QWs. ${ }^{4-22}$ Spin photocurrents at homogeneous excitation have been observed in $n$ and $p$-type quantum wells based on various semiconductor materials at very different types of optical excitation by application of laser radiation at spectral range from the visible to the very far-infrared (THz-frequencies).

In some optical experiments, where circularly polarized radiation is used to orient spins, the resulting spin photocurrent may represent a sum of spin-galvanic (SGE) and circular photogalvanic effects (CPGE). ${ }^{5,12} \mathrm{~A}$ characteristic feature of these spin photocurrents induced by circularly polarized radiation is that electric current it reverses its direction upon changing the radiation helicity from left-handed to right-handed and vice versa. A method which, on the one hand, provides a uniform distribution in spin subbands and, on the other hand, excludes the circular photogalvanic effect was introduced in Ref. 4. This method is based on the use of optical excitation and the assistance of an external magnetic field to achieve an in-plane polarization in (001)-grown low-dimensional structures. The spin-galvanic contribution was also experimentally extracted at pure optical excitation. ${ }^{5,12}$ In this experiments nonequilibrium spin orientation was obtained by resonant intersubband absorption of circularly polarized infrared radiation. The spectral behaviour of the SGE and the CPGE is qualitatively different. While SGE reproduces the spectral behaviour of inter-subband absorption coefficient, $K(\omega)$, the CPGE follows the derivation of $K(\omega)$ (see Refs. 5, 12, 23). Experiments on wavelength dependence of the photocurrent excited by inter-subband transitions were carried out making use of the tunability of the free-electron laser FELIX wavelength proved the spingalvanic effect at pure optical excitation. Experiments on spin-galvanic effect provide experimental access to spin properties of low-dimensional structures allowing us to determine the relative strength of the Rashba and Dresselhaus spin-splitting and spin relaxation times in semiconductor quantum wells. ${ }^{4,5,16}$

The observation of the inverse spin-galvanic effect demonstrates that electric current in non-magnetic but gyrotropic QWs results in a nonequilibrium spin polarization. The manipulation of the spin degree of freedom in electrically conducting systems by electric fields is at the heart of semiconductor spintronics. ${ }^{2}$ The feasibility to orient the spin of charge carriers in low-dimensional structures by driving 
an electric current through the device was theoretically predicted by Aronov and Lyanda-Geller ${ }^{24}$ and Edelstein ${ }^{25}$ nearly two decades ago. Just recently first direct experimental proofs of this effect were obtained in semiconductor QWs by Ganichev et al. ${ }^{26}$ and Silov et al. ${ }^{27}$ as well as in strained bulk material by Kato et al. ${ }^{28}$. Microscopically the effect is a consequence of spin-orbit coupling which lifts the spindegeneracy in $\boldsymbol{k}$-space together with spin dependent relaxation. ${ }^{\text {b }}$ Two microscopic mechanisms, namely scattering mechanism and precessional mechanism, based on Elliott-Yafet and D'yakonov-Perel' spin relaxation (for review see Ref. ${ }^{32}$ ), respectively, are responsible for the inverse spin-galvanic effect. Experimentally current induced spin orientation was investigated applying Faraday rotation and linearcircular dichroism in transmission of terahertz radiation, polarized luminescence, and space resolved Faraday rotation experiments in the near-infrared up to visible spectral range. ${ }^{26-35}$ Current induced spin orientation was detected in GaAs and InAs ${ }^{21}$ low-dimensional structures of various crystallographic orientation and in strained bulk InGaAs. We emphasize that the effect is observed even at technologically important room temperature. ${ }^{26,34,35}$ Variation of crystallographic direction demonstrated that, in agreement with the phenomenological theory, an in-plane electric current may result not only in the in-plane spin orientation but can also orient spins normal to the 2DEG's plane in (113)-grown structures.

\section{Spin-galvanic effect}

\subsection{Phenomenology}

A uniform nonequilibrium spin polarization obtained by any means yields a current, if the symmetry requirements, which allow $\boldsymbol{k}$-linear terms in the Hamiltonian, are met. Phenomenologically, an electric current can be linked to the electron's average nonequilibrium spin $\boldsymbol{S}$ by

$$
j_{\alpha}=\sum_{\gamma} Q_{\alpha \gamma} S_{\gamma}
$$

where $\boldsymbol{j}$ is an electric current density and $\boldsymbol{Q}$ is a second rank pseudotensor. Therefore in zinc-blende structure based QWs, nonzero components of $Q_{\alpha \gamma}$ exist in contrast to the corresponding bulk crystals. ${ }^{3}$ The nonzero components of the tensor $\boldsymbol{Q}$ depend on symmetry. Therefore angle between the nonequilibrium spin and the current changes in materials of different point symmetry. Spin-galvanic effect for different symmetries can be studied using samples grown on (001), (113) and (110) substrates. The point symmetries relevant for zinc-blende structure based QWs are $\mathrm{D}_{2 d}, \mathrm{C}_{2 v}$ and $\mathrm{C}_{s}$. For the sake of a simple description of the tensor equation we introduce

\footnotetext{
be note that more than twenty years ago Vorob'ev et al. ${ }^{29}$ observed a current induced spin polarization in bulk semiconductor (tellurium). This effect, theoretically predicted by Ivchenko and Pikus, ${ }^{30}$ is a consequence of the unique band structure of tellurium with hybridized spin-up and spin-down bands and is not related to spin relaxation. In zinc-blende structure based QWs this microscopic mechanism of the current induced spin polarization is absent. ${ }^{31}$
} 

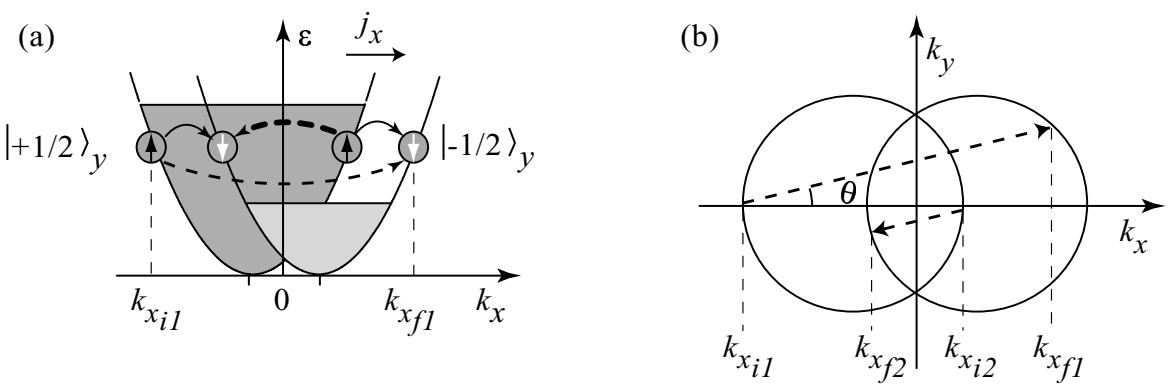

Fig. 1. Microscopic origin of the spin-galvanic current in the presence of $\boldsymbol{k}$-linear terms in the electron Hamiltonian. (a) One-dimensional sketch: the $\sigma_{y} k_{x}$ term in the Hamiltonian splits the conduction band into two parabolas with the spin $m_{s}= \pm 1 / 2$ pointing in the $y$-direction. If one spin subband is preferentially occupied, e.g., by spin injection (the $|+1 / 2\rangle_{y}$-states in the figure) asymmetric spin-flip scattering results in a current in the $x$-direction. The rate of spin-flip scattering depends on the value of the initial and final $\boldsymbol{k}$-vectors. Thus the transitions sketched by dashed arrows yield an asymmetric occupation of both subbands and hence a current flow. These transitions are also shown in two dimensions (b) by dashed arrows at scattering angle $\theta$. If instead of the spin-down subband the spin-up subband is preferentially occupied, the current direction is reversed. Model is given after Refs. 4, 7 .

two different Cartesian coordinate systems. For $\mathrm{D}_{2 d}$ and $\mathrm{C}_{2 v}$ symmetry, represented by symmetric and asymmetric QWs grown on (001)-oriented substrates, the tensor elements are given in the $(x y z)$ coordinate system:

$$
x\|[1 \overline{1} 0], \quad y\|[110], \quad z \|[001] .
$$

Here the coordinates $x$ and $y$ are in the reflection planes of both point groups; $z$ is along the growth direction normal to the plane of the $\mathrm{QW}$. The second coordinate system $\left(x y^{\prime} z^{\prime}\right)$ is convenient for (113)-grown samples representing $\mathrm{C}_{s}$ symmetry

$$
x\left\|[1 \overline{1} 0], \quad y^{\prime}\right\|[33 \overline{2}], \quad z^{\prime} \|[113] .
$$

In this case $x$ is normal to the only nonidentity symmetry element of $\mathrm{C}_{s}$, a mirror plane.

For (001)-grown asymmetric QWs which belongs to $\mathrm{C}_{2 v}$ symmetry only two linearly independent components, $Q_{x y}$ and $Q_{y x}$ may be nonzero so that

$$
j_{x}=Q_{x y} S_{y}, \quad j_{y}=Q_{y x} S_{x} .
$$

Hence, a spin polarization driven current needs a spin component lying in the plane of the QW. In $\mathrm{D}_{2 d}$ symmetry there is only one independent tensor component $Q_{x y}=Q_{y x}$. In $\mathrm{C}_{s}$ symmetry of (113)-oriented QWs an additional tensor component $Q_{x z^{\prime}}$ may be nonzero and the spin-galvanic current may be driven by nonequilibrium spins oriented normally to the plane of the QW.

Figure 1(a) illustrates the generation of a spin-galvanic current. As already addressed above, spin-galvanic current arises due to $\boldsymbol{k}$-linear terms in the effective Hamiltonian $\hat{H}=\sum_{l m} \beta_{l m} \sigma_{l} k_{m}$, where $\sigma_{l}$ are the Pauli spin matrices and $\beta_{l m}$ are 
real coefficients. The coefficients $\beta_{l m}$ form a pseudo-tensor subjected to the same symmetry restriction as the transposed pseudotensor $\boldsymbol{Q}$. The sources of $\boldsymbol{k}$-linear terms are the bulk inversion asymmetry (BIA) resulted in the Dresselhaus term ${ }^{36}$ (including a possible interface inversion asymmetry ${ }^{37}$ ), and a structural inversion asymmetry (SIA) causing the Rashba term. ${ }^{1}$ For a two-dimensional electron gas system, these terms lead to the situation sketched in Fig. 1(a). The electron energy band splits into two subbands which are shifted in $\boldsymbol{k}$-space, and each of the bands comprise states with spin up or down. The electron energy spectrum along $k_{x}$ with the spin-dependent term $\beta_{y x} \sigma_{y} k_{x}$ is shown. In this case $\sigma_{y}$ is a good quantum number. Spin orientation in the $y$-direction causes the unbalanced population in spin-down and spin-up subbands. As long as the carrier distribution in each subband is symmetric around the subband minimum at $k_{x_{ \pm}}$no current flows. The current flow is caused by $\boldsymbol{k}$-dependent spin-flip relaxation processes. Spins oriented in the $y$-direction are scattered along $k_{x}$ from the higher filled, e.g., spin subband $|+1 / 2\rangle_{y}$, to the less filled spin subband $|-1 / 2\rangle_{y}$. Four quantitatively different spin-flip scattering events exist and are sketched in Fig. 1(a) by bent arrows. The spin-flip scattering rate depends on the values of the wavevectors of the initial and the final states. ${ }^{32}$ Therefore spin-flip transitions, shown by solid arrows in Fig. 1(a), have the same rates. They preserve the symmetric distribution of carriers in the subbands and, thus, do not yield a current. However, the two scattering processes shown by broken arrows are inequivalent and generate an asymmetric carrier distribution around the subband minima in both subbands. This asymmetric population results in a current flow along the $x$-direction. Within this model of elastic scattering the current is not spin polarized since the same number of spin-up and spin-down electrons move in the same direction with the same velocity.

It must be pointed out that the above one-dimensional model, which in a clear way illustrates how a spin-galvanic current can occur, somehow simplifies the microscopic picture. The probability of the spin-flip processes $\left|+1 / 2, \boldsymbol{k}_{i}\right\rangle_{y} \rightarrow\left|-1 / 2, \boldsymbol{k}_{f}\right\rangle_{y}$ shown by arrows in Fig. 1(a) is given by the product $\left[v\left(\boldsymbol{k}_{i}-\boldsymbol{k}_{f}\right)\right]^{2}\left(\boldsymbol{k}_{f}+\boldsymbol{k}_{i}\right)^{2}$ (see Eq. (30) of Ref. 32). The amplitude $v\left(\boldsymbol{k}_{f}-\boldsymbol{k}_{i}\right)$ depends on $\boldsymbol{k}_{f}-\boldsymbol{k}_{i}$ and therefore the spin-flip process is asymmetric as needed for the occurrence of the current. However, for the one-dimensional model presented above the probability is given by $\left[v\left(k_{x_{f}}-k_{x_{i}}\right)\right]^{2}\left(k_{x_{f}}+k_{x_{i}}\right)^{2}$. In the case of elastic scattering, as sketched in Fig. 1(a), the magnitudes of the initial and final wavevectors are equal, $\left|k_{x_{i}}\right|=\left|k_{x_{f}}\right|$, thus $k_{x_{f}}+k_{x_{i}}=0$ and the probability of the spin-flip processes vanishes. A nonzero current is obtained for inelastic scattering and for elastic scattering with $k_{y} \neq 0$. The latter situation is depicted in Fig. 1(b).

\subsection{Spin-galvanic effect at optical orientation in the presence of a magnetic field}

The spin-galvanic effect can be investigated by pure optical spin orientation due to absorption of circularly polarized radiation in QWs. However, the irradiation 


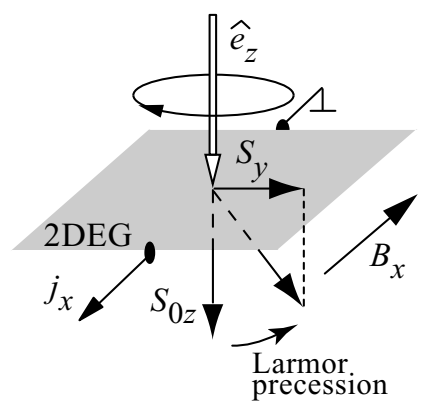

Fig. 2. Optical scheme of generating a uniform in-plane spin polarization which causes a spingalvanic current. Electron spins are oriented normal to the plane of QW by circularly polarized radiation and rotated into the plane by Larmor precession in an in-plane magnetic field $B_{x}$ (after Ref. 4).

of QWs with circularly polarized light also results in circular photogalvanic effect. Therefore a mixture of both effects may occur. The CPGE current is given by $j_{\lambda}=\sum_{\mu} \gamma_{\lambda \mu} \hat{e}_{\mu} E_{0}^{2} P_{\text {circ }}$, where $\boldsymbol{\gamma}$ is a pseudotensor, $P_{\text {circ }}, E_{0}$ and $\hat{\boldsymbol{e}}$ are radiation helicity, the electric field amplitude and the unit vector pointing in the direction of light propagation, respectively. Because of the tensor equivalence the irreducible components of $\boldsymbol{\gamma}$ and $\boldsymbol{Q}$ differ by a scalar factor. This fact results in the similar behaviour of both effects upon variation of optical experiment geometry, i.e. direction of light and current in respect to the crystallographic orientation. Microscopically, however, these two effect are crucially different. While the spin-galvanic effect may be caused by any means of spin injection, not only optical, the CPGE needs optical excitation with circularly polarized radiation. Even if the spin-galvanic effect is achieved by optical spin orientation the microscopic mechanisms of these two effects are different. The spin-galvanic effect is caused by asymmetric spin-flip scattering of spin polarized carriers and it is determined by the process of spin relaxation. If spin relaxation is absent, the spin-galvanic current vanishes. In contrast, the CPGE is the result of selective photoexcitation of carriers in $\boldsymbol{k}$-space with circularly polarized light due to optical selection rules and depends on momentum relaxation.

In this section we will describe a method which, on the one hand, achieves a uniform distribution in spin subbands by optical means and, on the other hand, excludes the circular photogalvanic effect. This method was introduced in Ref. 4. Spin polarization is obtained by absorption of circularly polarized radiation at normal incidence on (001)-grown QWs as depicted in Fig. 2. For normal incidence the spin-galvanic effect as well as CPGE vanish because $S_{x}=S_{y}=0$ (see Eqs. (4)) and $\hat{e}_{x}=\hat{e}_{y}=0$. Thus, a spin orientation $S_{0 z}$ along the $z$-coordinate is achieved but no spin photocurrent is obtained.

The steady-state spin polarization $S_{0 z}$ is proportional to the spin generation rate. An in-plane component of the spins, necessary for the spin-galvanic effect, is generated applying a magnetic field $\boldsymbol{B} \| x$. The field perpendicular to both, the light 


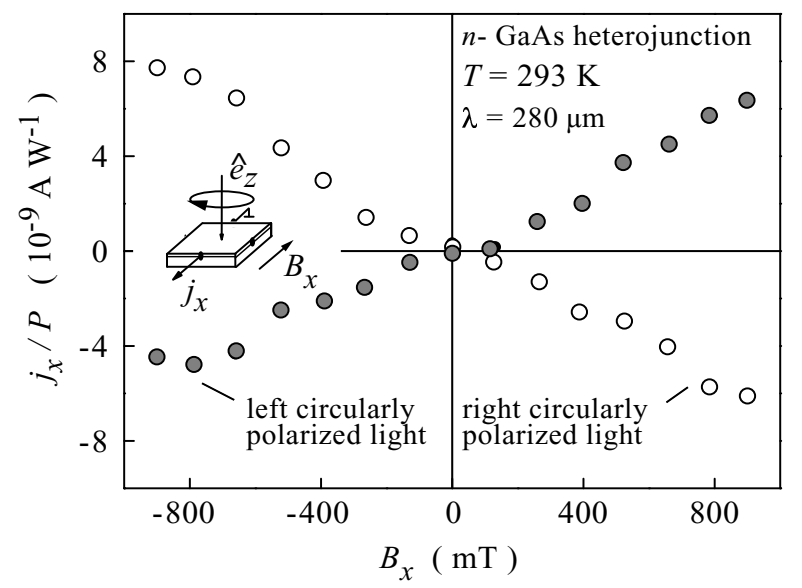

Fig. 3. Magnetic field dependence of the spin-galvanic current normalized by $P$ achieved by intrasubband transitions within the $e 1$ conduction subband by excitation with radiation of $\lambda=280 \mu \mathrm{m}$ wavelength. Results are plotted for an (001)-grown GaAs single heterojunction at room temperature. The inset shows the geometry of the experiment where $\hat{e}_{z}$ indicates the direction of the incoming light. Data are given after Ref. 7.

propagation direction $\hat{e}_{z}$ and the optically oriented spins, rotates the spins into the plane of the 2DEG due to Larmor precession. A nonequilibrium spin polarization $S_{y}$ is given by

$$
S_{y}=-\frac{\omega_{L} \tau_{s \perp}}{1+\left(\omega_{L} \tau_{s}\right)^{2}} S_{0 z}
$$

where $\tau_{s}=\sqrt{\tau_{s \|} \tau_{s \perp}}, \tau_{s \|}, \tau_{s \perp}$ are the longitudinal and transverse electron spin relaxation times, and $\omega_{L}$ is the Larmor frequency. The denominator in Eq. (5), which yields a decay of $S_{y}$ for $\omega_{L}$ exceeding the inverse spin relaxation time, is well known from the Hanle effect. ${ }^{38,39}$

Using this method the spin-galvanic effect has been experimentally investigated on $n$-type GaAs and InAs samples (Figs. 3-6). Figure 3 shows the spin-galvanic current as a function of the external magnetic field. For low magnetic field strengths $B$, where $\omega_{L} \tau_{s}<1$ holds, the photocurrent increases linearly with $B$ as given in Eqs. (4) and (5). This is seen in the room temperature data of Fig. 3 as well as in the $4.2 \mathrm{~K}$ data in Fig. 4 for $B \leq 1 \mathrm{~T}$. The polarity of the current depends on the direction of the excited spins (see Figs. 3 and 4, parallel or antiparallel to the $z$ direction for right or left circularly polarized light, respectively) and on the direction of the applied magnetic field (see Figs. $3-5, \pm B_{x}$-directions). For a magnetic field pointing along $\langle 110\rangle$ the current is parallel (antiparallel) to the magnetic field vector. For $\boldsymbol{B} \|\langle 100\rangle$ both the transverse and the longitudinal effects are observed. ${ }^{7}$

For higher magnetic fields the current assumes a maximum and decreases upon further increase of $B$, as shown in Fig. 4. This drop of the current is ascribed to the Hanle effect. ${ }^{38}$ The experimental data are well described by Eqs. (4) and (5). 


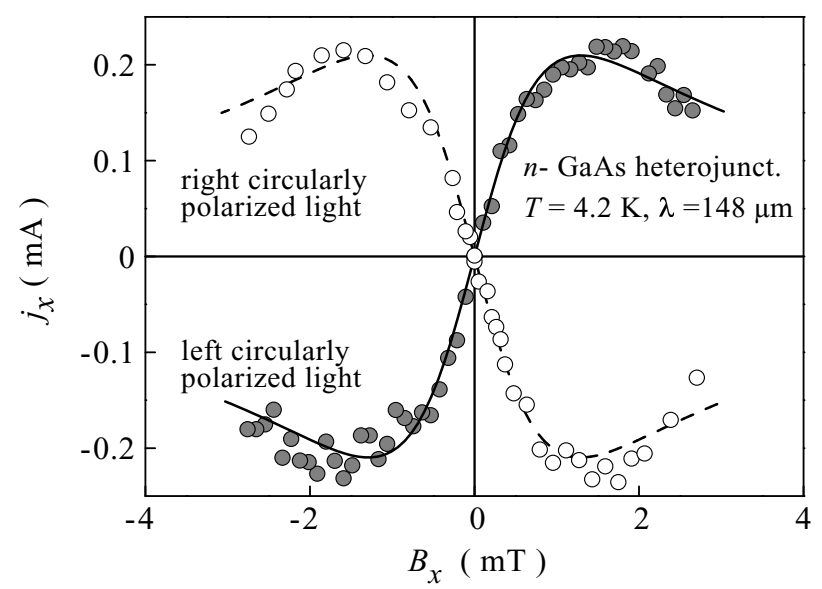

Fig. 4. Spin-galvanic current $j_{x}$ as a function of magnetic field $B$ for normally incident righthanded (open circles) and left-handed (solid circles) circularly polarized radiation at $\lambda=148 \mu \mathrm{m}$ and radiation power $20 \mathrm{~kW}$. Measurements are presented for an $n$-type GaAs/AlGaAs single heterojunction at $T=4.2 \mathrm{~K}$. Solid and dashed curves are fitted after Eqs. (4) and (5) using the same value of the spin relaxation time $\tau_{s}$ and scaling of the ordinate. Data are given after Ref. 4 .

The observation of the Hanle effect demonstrates that free carrier intra-subband transitions can polarize the spins of electron systems. The measurements allow us to obtain the spin relaxation time $\tau_{s}$ from the peak position of the photocurrent where $\omega_{L} \tau_{s}=1$ holds. ${ }^{4}$

In $p$-GaAs QWs at terahertz excitation causing spin polarization of holes only, no spin-galvanic effect has been detected. ${ }^{4,40}$ The spin-galvanic effect in $p$-type material for inter- or intra-subband excitation could not be observed because of the experimental procedure which makes use of the Larmor precession to obtain an inplane spin polarization. This is due to the fact that the in-plane $g$-factor for heavy holes is very small ${ }^{41}$ which makes the effect of the magnetic field negligible. ${ }^{4}$ This result does not exclude the spin-galvanic effect in $p$-type materials which might be observable by generation of nonequilibrium spin polarization in the proper direction, for instance in the plane of (001)-grown QWs, applying optical excitation or by means of hole injection.

On a phenomenological level and for low magnetic fields, $\omega_{L} \tau_{s} \ll 1$, this magnetic field induced spin photocurrent can be described by

$$
j_{\alpha}=\sum_{\beta \gamma} \mu_{\alpha \beta \gamma} B_{\beta} i\left(\boldsymbol{E} \times \boldsymbol{E}^{*}\right)_{\gamma}=\sum_{\beta \gamma} \mu_{\alpha \beta \gamma} B_{\beta} \hat{e}_{\gamma} E^{2} P_{\text {circ }},
$$

where $\mu_{\alpha \beta \gamma}$ is a third-rank tensor. As $P_{\text {circ }}$ is a pseudoscalar and $\boldsymbol{B}$ a pseudovector, $\mu_{\alpha \beta \gamma}$ is a regular negative-parity third-rank tensor which is allowed in inversion asymmetric structures only. Gyrotropy at zero magnetic field, as in the case of only the optical excited spin-galvanic effect or of the circular photogalvanic effect, is not necessary. We note that in nongyrotropic $p$-type bulk GaAs a magnetic field induced 


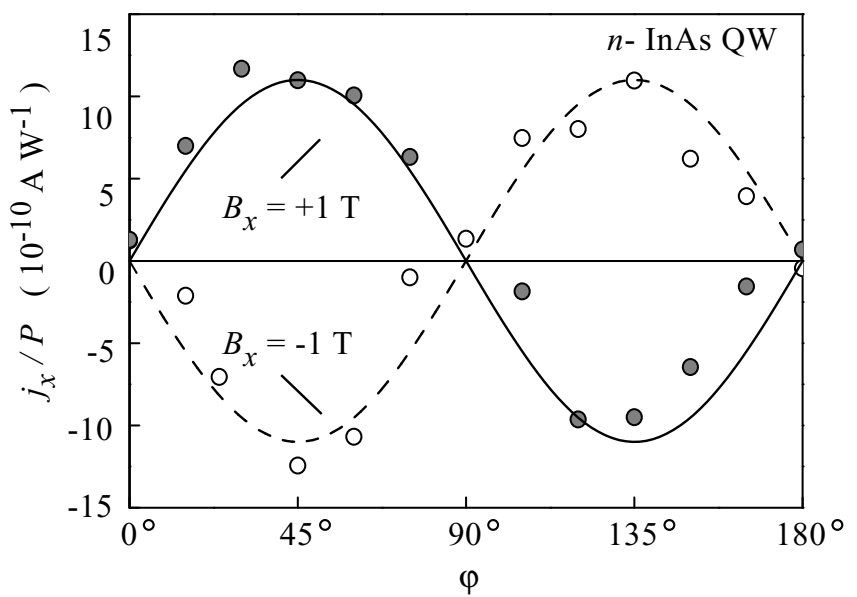

Fig. 5. Spin-galvanic current normalized by $P$ as a function of the phase angle $\varphi$ in an (001)grown $n$-type InAs QW of $15 \mathrm{~nm}$ width at $T=4.2 \mathrm{~K}$. The photocurrent excited by normal incident radiation of $\lambda=148 \mu \mathrm{m}$ is measured in the $x$-direction parallel (full circles) and antiparallel (open circles) to the in-plane magnetic field $B_{x}$. Solid and dashed curves are fitted after Eqs. (7) using the same scaling of the ordinate. Data are given after Ref. 7.

circular photogalvanic effect was previously observed under intra-band excitation. ${ }^{42}$ However, this effect is not due to spin orientation and does not occur in $p$-type QWs due to spatial quantization. ${ }^{43}$ In QWs under normal incidence of the light and for a magnetic field lying in the plane of a $\mathrm{QW}$ of $\mathrm{C}_{2 v}$ symmetry, which corresponds to the measurements on (001)-grown QWs, the current is described by two independent components of the tensor $\boldsymbol{\mu}$ and can be written as

$$
j_{x}=\mu_{x x z} B_{x} \hat{e}_{z} E^{2} P_{\text {circ }}, \quad j_{y}=\mu_{y y z} B_{y} \hat{e}_{z} E^{2} P_{\text {circ }} .
$$

The current $\boldsymbol{j}$ and the magnetic field $\boldsymbol{B}$ are parallel (or antiparallel) when the magnetic field is applied along $\langle 110\rangle$ and neither parallel nor perpendicular for $\boldsymbol{B} \|\langle 100\rangle$. In $\mathrm{D}_{2 d}$ symmetry QWs with symmetric interfaces $\mu_{x x z}=-\mu_{y y z}$ and therefore the current is perpendicular to the magnetic field for $\boldsymbol{B} \|\langle 100\rangle$. These equations describe well the anisotropy as well as the helicity dependence of the spin-galvanic effect shown in Fig. 5.

For optical excitation of the spin-galvanic effect mid-infrared (MIR), far-infrared (THz-frequencies) and visible laser radiation was used. ${ }^{5-7}$ Most of the measurements were carried out in the long wavelength range with photon energies less than the energy gap of investigated semiconductors. For investigations of spin photocurrents infrared excitation has several advantages. First of all below the energy gap the absorption is very weak and therefore allows homogeneous excitation of the $2 \mathrm{D}$ electron gas. Furthermore, in contrast to inter-band excitation resulting in the valence band - conduction band transition, there are no spurious photocurents due to other mechanisms like the Dember effect, photovoltaic effects at contacts and 


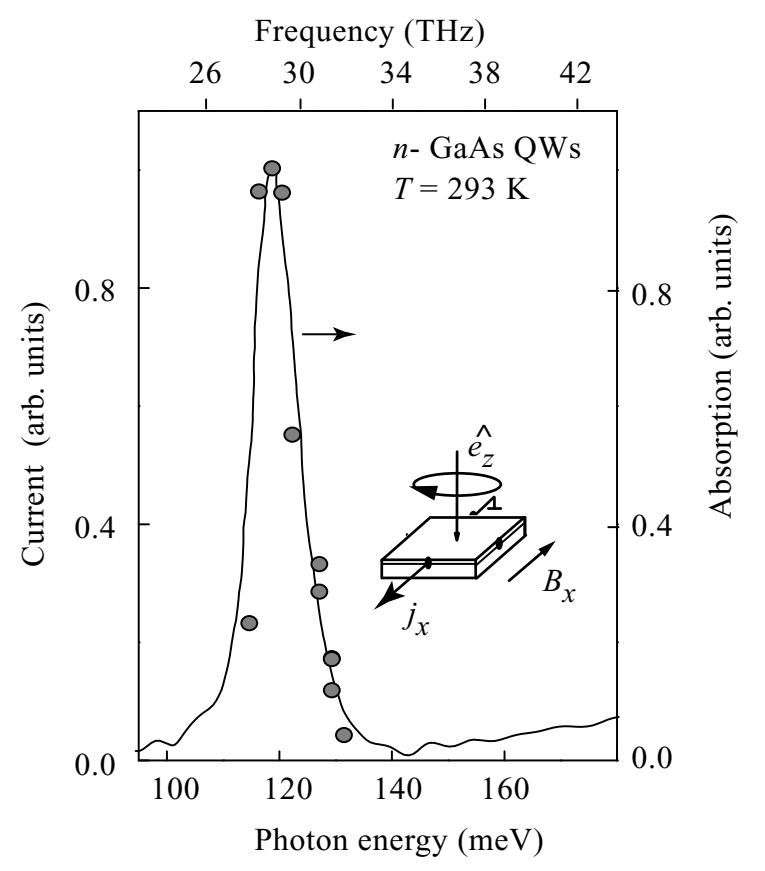

Fig. 6. Spectral dependence of the spin-galvanic current in (001)-grown $n$-type GaAs QWs of $8.2 \mathrm{~nm}$ width at room temperature. Data (circles) are presented for optical excitation at normal incidence of right-handed circularly polarized radiation. A magnetic field of $B_{x}=1 \mathrm{~T}$ was applied. For comparison the absorption spectrum is shown by the full line. Data are given after Ref. 45 .

Schottky barriers etc. To avoid this problem in the measurements applying visible radiation the current was recorded by a lock-in amplifier in phase with the photoelastic modulator. ${ }^{4}$

In the MIR-terahertz range spin-galvanic currents have been recorded for intersubband as well as for intra-subband transitions. ${ }^{4,12,40}$ Direct inter-subband transitions have been achieved in GaAs QWs of $8.2 \mathrm{~nm}$ and $8.6 \mathrm{~nm}$ widths in absorption of radiation in the range of $9 \mu \mathrm{m}$ to $11 \mu \mathrm{m}$ wavelength. ${ }^{44,45}$ The wavelength dependence of the spin-galvanic effect obtained in this spectral range repeats the spectral behaviour of direct inter-subband absorption (see Fig. 6). This observation is in agreement with the mechanism of the spin-galvanic effect and the microscopic theory given below in section 1.4. The occurrence of a spin-galvanic current requires only a spin polarization in the lower subband and asymmetric spin relaxation. In the present case the spin orientation is generated by resonant spin-selective optical excitation followed by spin-nonspecific thermalization. Therefore the magnitude of the spin polarization and hence the current depends on the absorption strength. The spin-galvanic effect due to indirect transitions has been obtained in $n$-type GaAs and InAs QWs applying THz radiation of pulsed molecular laser operating at wavelength from $77 \mu \mathrm{m}$ up to $496 \mu \mathrm{m}$ (see Figs. $3-5$ ). 
The presence of the MIR and terahertz radiation excited spin-galvanic effect, which is due to spin orientation, gives clear evidence that direct inter-subband and Drude absorption of circularly polarized radiation results in a monopolar spin orientation. Mechanisms of the monopolar spin orientation were introduced in Refs. 4446. We would like to emphasize that spin-sensitive $e 1-e 2$ inter-subband transitions in (001)-grown $n$-type QWs have been observed at normal incidence when there is no component of the electric field of the radiation normal to the plane of the QWs. Generally it is believed that inter-subband transitions in $n$-type QWs can only be excited by radiation polarized in the growth direction $z$ of the QWs. ${ }^{6,47}$ Furthermore such transitions are spin insensitive and, hence, do not lead to optical orientation. Since the argument, leading to these selection rules, is based on the effective mass approximation in a single-band model, the selection rules are not rigorous. The theory of the mechanism, which leads to spin orientation in this geometry, was recently developed in Ref. 46.

\subsection{Spin-galvanic effect at pure optical excitation}

A spin-galvanic effect at optical excitation may be observed if an in-plane component of the spin polarization is present due to oblique incidence of the exciting circularly polarized radiation. As addressed above, in this case, however, the circular photogalvanic effect may also occur interfering with the spin-galvanic effect. Nevertheless, a spin-galvanic current without an admixture of the CPGE can be obtained at inter-subband transitions in $n$-type GaAs QWs. ${ }^{12}$

The spin-galvanic effect at optical excitation has been detected by making use of the spectral tunability of the free-electron laser FELIX. ${ }^{48}$ Figure 7 shows the photon energy dependence of the current measured for incidence of radiation in two different planes with components of propagation along the $x$ - and $y$-directions. In this figure the photocurrent due to $\sigma_{+}$irradiation is compared to the absorption spectrum. It can be seen that for a current along $y \|[110]$ the spectral shape follows closely the absorption spectrum. This spectral behaviour of the current indicates optically induced spin-galvanic current which is proportional to the absorbance (Eqs. (13)) and, hence, assumes a maximum at resonance. ${ }^{7,12}$ When in the same experiment the sample was rotated by $90^{\circ}$ around $z$ the sign change in the current, now along $x \|[1 \overline{1} 0]$, the spectral shape is similar to the derivative of the absorption spectrum. In particular, there is a change of sign which occurs at the line center of the absorption. As shown in Ref. 23, such a spectral behaviour is characteristic for the CPGE which changes sign and vanishes in the center of the resonance.

The fact that the current in the $x$-direction is dominated by CPGE and in the $y$ direction by the spin-galvanic effect is caused by the crystallographic nonequivalence of the axes [110] and [110] in asymmetric (001)-grown QWs. Both currents, CPGE and the spin-galvanic current, are due to spin splitting of subbands in $\boldsymbol{k}$-space. This spin splitting is very different for the $x$ - and $y$-directions due to the interplay of BIA and SIA terms in the Hamiltonian when rotating the wavevector in the QW plane. 


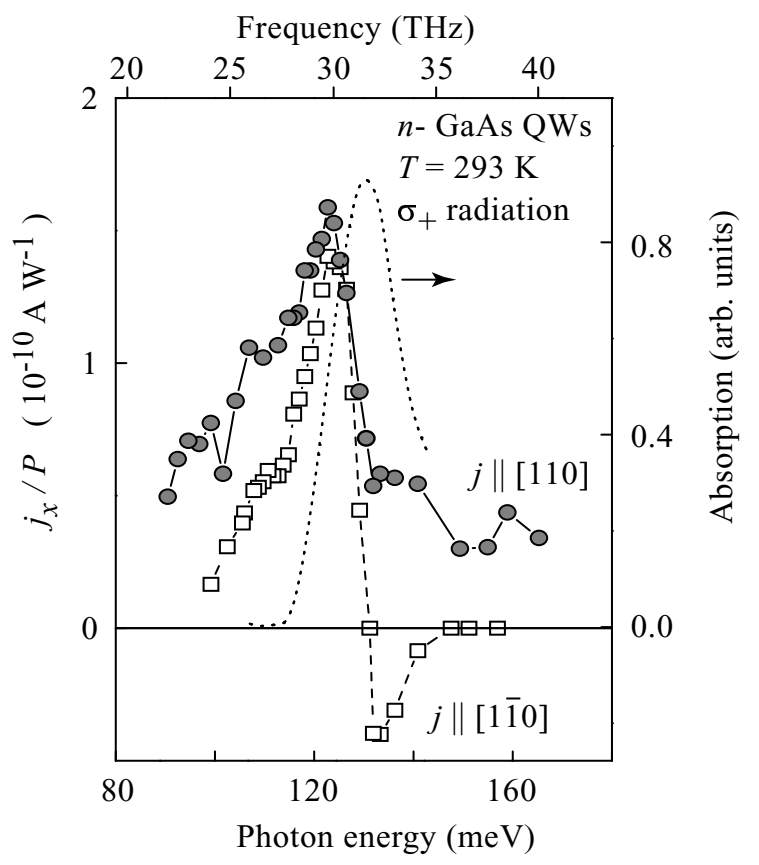

Fig. 7. Photocurrent in QWs normalized by the light power $P$ at oblique incidence of righthanded circularly polarized radiation on $n$-type (001)-grown GaAs/AlGaAs QWs of $8.2 \mathrm{~nm}$ width at $T=293 \mathrm{~K}$ as a function of the photon energy $\hbar \omega$. Circles: current detected in [110] direction in

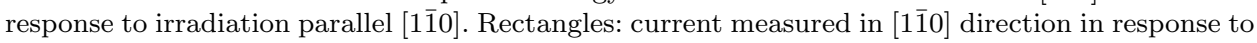
irradiation parallel [110]. The dotted line shows the absorption measured using a Fourier transform spectrometer. Data are given after Ref. 12 .

The pseudotensors $\gamma$ and $\boldsymbol{Q}$ determining the current are related to the transposed pseudotensor $\boldsymbol{\beta}$. They are subjected to the same symmetry restrictions so that their irreducible components differ only by scalar factors. In $\mathrm{C}_{2 v}$ symmetry $\beta_{y x} \neq \beta_{x y}$, and it is reasonable to introduce symmetric and antisymmetric components $\beta_{\mathrm{BIA}}^{(\nu)}=\left(\beta_{x y}^{(\nu)}+\beta_{y x}^{(\nu)}\right) / 2$ and $\beta_{\mathrm{SIA}}^{(\nu)}=\left(\beta_{x y}^{(\nu)}-\beta_{y x}^{(\nu)}\right) / 2$, where $\nu=1,2$ indicates the $e 1$ and $e 2$ subbands, respectively. $\beta_{\mathrm{BIA}}^{(\nu)}$ and $\beta_{\mathrm{SIA}}^{(\nu)}$ result from bulk inversion asymmetry and from structural inversion asymmetry, correspondingly.

As it is shown in Refs. 4 and 8 (for reviews see also Refs $5^{-7}$ ) and sketched in Fig. 8, both the spin-galvanic and the CPGE currents, say in the $x$-direction, are caused by band splitting in the $k_{y}$-direction and, therefore, are proportional to $\beta_{y x}$ (see Fig. 8). Note that for current in the $y$-direction one should interchange the indices $x$ and $y$. Then the currents in the $x$ - and $y$-directions are

$$
\begin{aligned}
j_{x} & =A_{\mathrm{CPGE}}\left[\left(\beta_{\mathrm{BIA}}^{(1)}-\beta_{\mathrm{SIA}}^{(1)}\right)-\left(\beta_{\mathrm{BIA}}^{(2)}-\beta_{\mathrm{SIA}}^{(2)}\right)\right] P_{\mathrm{circ}} \hat{e}_{y} \\
& +A_{\mathrm{SGE}}\left(\beta_{\mathrm{BIA}}^{(1)}-\beta_{\mathrm{SIA}}^{(1)}\right) S_{y}, \\
j_{y} & =A_{\mathrm{CPGE}}\left[\left(\beta_{\mathrm{BIA}}^{(1)}+\beta_{\mathrm{SIA}}^{(1)}\right)-\left(\beta_{\mathrm{BIA}}^{(2)}+\beta_{\mathrm{SIA}}^{(2)}\right)\right] P_{\mathrm{circ}} \hat{e}_{x}
\end{aligned}
$$


(a)

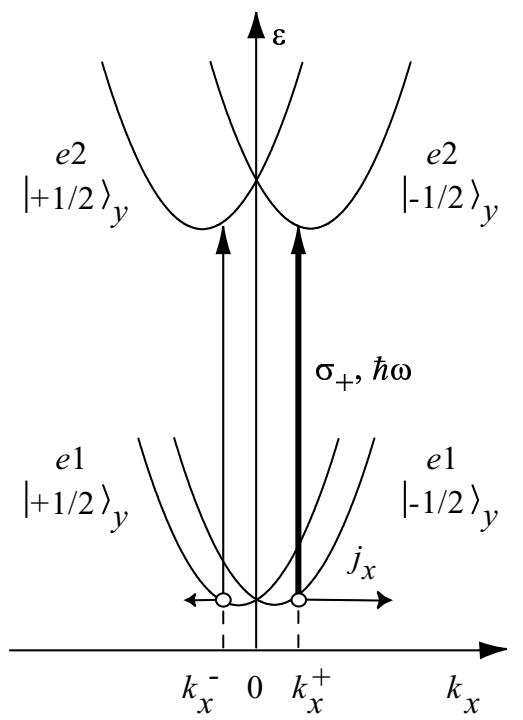

(b)

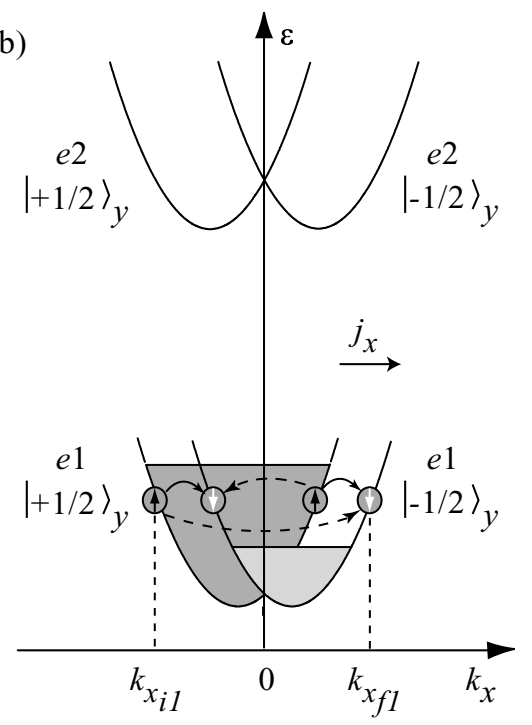

Fig. 8. Microscopic picture of (a) circular photogalvanic effect and (b) spin-galvanic effect for inter-subband excitation in $\mathrm{C}_{2 v}$ point group samples at oblique incidence. In (a) the current $j_{x}$ is caused by the imbalance of optical transition probabilities at $k_{x}^{-}$and $k_{x}^{+}$decaying with the momentum relaxation time $\tau_{p}$. Excitation with $\sigma_{+}$radiation of $\hbar \omega$ less than the energy subband separation $\varepsilon_{21}$ at $k=0$ induces direct spin-conserving transitions (vertical arrows) at $k_{x}^{-}$and $k_{x}^{+}$. The rates of these transitions are different as illustrated by the different thickness of the arrows. This leads to a photocurrent due to an asymmetric distribution of carriers in $\boldsymbol{k}$-space. Increasing of the photon energy shifts more intensive transitions to the left and less intensive to the right resulting in a current sign change. In (b) the current occurs after thermalization in the lowest subband which results in the spin orientation in the $e 1$ subband. This spin-galvanic current is caused by asymmetric spin-flip scattering. The rate of spin-flip scattering depends on the value of the initial and final $\boldsymbol{k}$-vectors. Thus transitions sketched by dashed arrows yield an asymmetric occupation of both subbands and hence a current flow which decays with the spin relaxation time $\tau_{s}$. The magnitude of the spin polarization and hence the current depends on the initial absorption strength but not on the momentum $\boldsymbol{k}$ of initial optical transition. Therefore the shape of the spectrum of the spin-galvanic current follows the absorption. After Ref. 12.

$$
+A_{\mathrm{SGE}}\left(\beta_{\mathrm{BIA}}^{(1)}+\beta_{\mathrm{SIA}}^{(1)}\right) S_{x}
$$

where $A_{\mathrm{CPGE}}$ and $A_{\mathrm{SGE}}$ contain all scalar parameters, including the intensity, and the scalars relating to the irreducible components of $\gamma$ and $\boldsymbol{Q}$, respectively. The subscripts CPGE and SGE indicate the circular photogalvanic effect and the spingalvanic effect, respectively.

In the present case the spin polarization $\boldsymbol{S}$ is obtained by optical orientation, its sign and magnitude are proportional to $P_{\text {circ }}$ and it is oriented along the in-plane component of $\hat{\boldsymbol{e}}$. The magnitude of CPGE is determined by the values of $\boldsymbol{k}$ in the initial and final states, and hence depends on the spin splitting $\beta_{\text {BIA }}$ and $\beta_{\text {SIA }}$ of both $e 1$ and $e 2$ subbands. In contrast, the spin-galvanic effect is due to relaxation between the spin states of the lowest subband and hence depends only on $\beta_{\text {BIA }}$ and 
$\beta_{\text {SIA }}$ of the lowest subband.

The above equations show that in directions $x$ and $y$ the spin-galvanic current and the CPGE are proportional to expressions with the difference and the sum of BIA and SIA terms, respectively. The relative strengths of BIA and SIA terms of subbands depend on the details of the structural properties of QWs. For the data of Fig. 7 it appears that in the case where the BIA and SIA contributions add, the spin-galvanic effect dominates over the CPGE consistent with the lack of sign change for the current along the $y$-direction. Conversely when BIA and SIA terms subtract the spin-galvanic effect is suppressed and the CPGE dominates. Hence, at the maximum of absorption, where the CPGE is equal to zero for both directions, the current obtained is caused solely by the spin-galvanic effect.

\subsection{Microscopic theory of spin-galvanic effect at resonant inter-subband absorption}

The microscopic theory of the spin-galvanic effect for inter-subband transitions in $n$-type zinc-blende structure based QWs of $\mathrm{C}_{2 v}$ symmetry has been developed by Ivchenko and Tarasenko in Ref. 12. In this case the spin orientation (see Fig. 8(b)) is generated by resonant spin-selective optical excitation (see Fig. 8(a)) followed by spin-nonspecific thermalization. As it has been addressed above optical excitation besides spin orientation causes the circular photogalvanic effect (see Fig. 8(a)). The theory of this effect is developed in Ref. 23 and is out of scope of the present paper.

The occurrence of a current is due to the spin dependence of the electron scattering matrix elements $\hat{M}_{\boldsymbol{k}^{\prime}, \boldsymbol{k}}$. The $2 \times 2$ matrix $\hat{M}_{\boldsymbol{k}^{\prime}, \boldsymbol{k}}$ can be written as a linear combination of the unit matrix $\hat{I}$ and Pauli matrices $\sigma_{\alpha}$ as follows

$$
\hat{M}_{\boldsymbol{k}^{\prime}, \boldsymbol{k}}=A_{\boldsymbol{k}^{\prime}, \boldsymbol{k}} \hat{I}+\boldsymbol{\sigma} \cdot \boldsymbol{B}_{\boldsymbol{k}^{\prime}, \boldsymbol{k}},
$$

where $A_{\boldsymbol{k}^{\prime}, \boldsymbol{k}}^{*}=A_{\boldsymbol{k}, \boldsymbol{k}^{\prime}}, B_{\boldsymbol{k}^{\prime} \boldsymbol{k}}^{*}=B_{\boldsymbol{k}, \boldsymbol{k}^{\prime}}$ due to hermiticity of the interaction and $A_{-\boldsymbol{k}^{\prime},-\boldsymbol{k}}=A_{\boldsymbol{k} \boldsymbol{k}^{\prime}}, B_{-\boldsymbol{k}^{\prime},-\boldsymbol{k}}=-B_{\boldsymbol{k}, \boldsymbol{k}^{\prime}}$ due to the symmetry under time inversion. The spin-dependent part of the scattering amplitude in (001)-grown QW structures is given by ${ }^{32}$

$$
\boldsymbol{\sigma} \cdot \boldsymbol{B}_{\boldsymbol{k}^{\prime}, \boldsymbol{k}}=v\left(\boldsymbol{k}-\boldsymbol{k}^{\prime}\right)\left[\sigma_{x}\left(k_{y}^{\prime}+k_{y}\right)-\sigma_{y}\left(k_{x}^{\prime}+k_{x}\right)\right] .
$$

We note that Eq. (11) determines the spin relaxation time, $\tau_{s}^{\prime}$, due to the ElliottYafet mechanism. The spin-galvanic current has the form ${ }^{12}$

$$
\begin{aligned}
& j_{\mathrm{SGE}, x}=Q_{x y} S_{y} \sim e n_{s} \frac{\beta_{y x}^{(1)}}{\hbar} \frac{\tau_{p}}{\tau_{s}^{\prime}} S_{y}, \\
& j_{\mathrm{SGE}, y}=Q_{y x} S_{x} \sim e n_{s} \frac{\beta_{x y}^{(1)}}{\hbar} \frac{\tau_{p}}{\tau_{s}^{\prime}} S_{x} .
\end{aligned}
$$

Since scattering is the origin of the spin-galvanic effect, the current $j_{\mathrm{SGE}}$ is determined by the Elliott-Yafet spin relaxation process even if other spin relaxation mechanisms dominate. The Elliott-Yafet relaxation time $\tau_{s}^{\prime}$ is proportional to the 
momentum relaxation time $\tau_{p}$. Therefore the ratio $\tau_{p} / \tau_{s}^{\prime}$ in Eq. (12) does not depend on the momentum relaxation time. The in-plane average spin, e.g., $S_{x}$, in Eq. (12) decays with the total spin relaxation time $\tau_{s}$. Thus the time decay of the spin-galvanic current following pulsed photoexcitation is determined by $\tau_{s}$. This time may have contributions from any spin relaxing process and in the present case of GaAs QWs is determined by the D'yakonov-Perel' mechanism.

For the case where spin relaxation is obtained as a result of inter-subband absorption of circularly polarized radiation, the current is given by

$$
\begin{aligned}
& j_{\mathrm{SGE}, x}=Q_{x y} S_{y} \sim e \frac{\beta_{y x}^{(1)}}{\hbar} \frac{\tau_{p} \tau_{s}}{\tau_{s}^{\prime}} \frac{\eta_{12} I}{\hbar \omega} P_{\text {circ }} \xi \hat{e}_{y}, \\
& j_{\mathrm{SGE}, y}=Q_{y x} S_{x} \sim e \frac{\beta_{x y}^{(1)}}{\hbar} \frac{\tau_{p} \tau_{s}}{\tau_{s}^{\prime}} \frac{\eta_{12} I}{\hbar \omega} P_{\text {circ }} \xi \hat{e}_{x},
\end{aligned}
$$

where $\eta_{12}$ is the absorbance at the transitions between the $e 1$ and $e 2$ subbands. The parameter $\xi$ varying between 0 and 1 is the ratio of photoexcited electrons relaxing to the $e 1$ subband with and without spin-flip. It determines the degree of spin polarization in the lowest subband (see Fig. 8(b)) and depends on the details of the relaxation mechanism. Optical orientation requires $\xi \neq 0$ (see Refs. 38, 49). Equations (13) show that the spin-galvanic current is proportional to the absorbance and is determined by the spin splitting in the first subband, $\beta_{y x}^{(1)}$ or $\beta_{x y}^{(1)}$.

\section{Spin orientation by current (inverse spin-galvanic effect)}

Phenomenologically, electron's averaged nonequilibrium spin can be linked to the an electric current by

$$
S_{\alpha}=\sum_{\gamma} R_{\alpha \gamma} j_{\gamma}
$$

where $\boldsymbol{R}$ is a second rank pseudotensor which irreducible components differ from that of $\boldsymbol{Q}$ by a scalar factor due to the tensor equivalence.

Microscopic picture of this effect is sketched in Fig. 9(b) for hole gas in structures of $\mathrm{C}_{s}$-symmetry, a situation relevant for the experiments of Refs. $26,33,34$. In the simplest case the electron's (or hole's) kinetic energy in a quantum well depends quadratically on the in-plane wavevector components $k_{x}$ and $k_{y}$. In equilibrium, the spin degenerated $k_{x}$ and $k_{y}$ states are symmetrically occupied up to the Fermi energy $E_{F}$. If an external electric field is applied, the charge carriers drift in the direction of the resulting force. The carriers are accelerated by the electric field and gain kinetic energy until they are scattered (Fig. 9(a)). A stationary state forms where the energy gain and the relaxation are balanced resulting in a non-symmetric distribution of carriers in $\boldsymbol{k}$-space. The holes acquire the average quasi-momentum

$$
\langle\boldsymbol{k}\rangle=\frac{e \tau_{p}}{\hbar} \boldsymbol{E}=\frac{m^{*}}{e \hbar p} \boldsymbol{j},
$$

where $\boldsymbol{E}$ is the electric field strength, $\tau_{p}$ the momentum relaxation time, $\boldsymbol{j}$ the electric current density, $m^{*}$ the effective mass, $p$ the hole concentration and $e$ the 
elementary charge. As long as spin-up and spin-down states are degenerated in $\boldsymbol{k}$ space the energy bands remain equally populated and a current is not accompanied by spin orientation. In QWs made of zinc-blende structure material like GaAs, however, the spin degeneracy is lifted due to lack of inversion symmetry and lowdimensional quantization, ${ }^{1,36}$ (for recent reviews see Refs. 5-7 and 50-52) dispersion reads

$$
\varepsilon=\frac{\hbar^{2} \boldsymbol{k}^{2}}{2 m^{*}}+\beta_{l m} \sigma_{l} k_{m}
$$

with the spin-orbit pseudotensor $\boldsymbol{\beta}$ and the Pauli spin matrices $\sigma_{l}$. The parabolic energy band splits into two subbands of opposite spin directions shifted in $\boldsymbol{k}$-space symmetrically around $\boldsymbol{k}=0$ with minima at $\pm k_{0}$. The corresponding dispersion is sketched in Fig. 9(b). To be specific for the coupling constant $\beta$ and the mechanism depicted in Fig. 9(b) we consider solely spin-orbit interaction due to a Hamiltonian of the form $H_{S O}=\beta \sigma_{z}^{\prime} k_{x}$. This corresponds to a subband splitting for eigenstates with spins pointing in $z^{\prime}$-direction, normal to the quantum well plane and detectable in experiment. In our QWs of $\mathrm{C}_{s}$ symmetry the $x$-direction lies along [110] in the QW plane. In the presence of an in-plane electric field the $\boldsymbol{k}$-space distribution of carriers gets shifted yielding an electric current. Due to the band splitting carrier relaxation becomes spin dependent. Relaxation processes including spin flips are different for the two subbands because the quasi-momentum transfer from initial to final states is different. ${ }^{32}$ In Fig. 9(b) the $\boldsymbol{k}$-dependent spin-flip scattering processes are indicated by arrows of different lengths and thicknesses. As a consequence different numbers of spin-up and spin-down carriers contribute to the current causing a stationary spin orientation. In this picture we assume that the origin of the current induced spin orientation is, as sketched in Fig. 9(b), exclusively due to scattering and hence dominated by the Elliott-Yafet spin relaxation process (relaxation mechanism).

The other possible mechanism resulting in the current induced spin orientation is based on the D'yakonov-Perel spin relaxation. So far we assumed that in structures of $\mathrm{C}_{s}$ symmetry the subband spin splitting occurs for spin eigenstates pointing normal to the QW. However, if the hole subbands are also split due to a spin-orbit coupling of the type $\propto \sigma_{x} k_{y}$ in the Hamiltonian an additional mechanism of spin orientation, the precession one, ${ }^{24,31}$ needs to be taken into account. The difference in the spin relaxation rates for spin-up and spin-down subbands is now determined by the D'yakonov-Perel spin relaxation process. In this case the relaxation rate depends on the average $\boldsymbol{k}$-vector, ${ }^{36}$ equal to $\bar{k}_{3 / 2}=-k_{0}+\langle k\rangle$ for the spin-up and $\bar{k}_{-3 / 2}=k_{0}+\langle k\rangle$ for the spin-down subband. Hence also for the D'yakonov-Perel spin relaxation mechanism a current through the hole gas causes spin orientation (precessional mechanism).

Spin orientation by electric current in low-dimensional structures is observed applying various experimental techniques, comprising transmission of polarized THzradiation, polarized luminescence and space resolved Faraday rotation. ${ }^{26-35,21}$ Here we briefly sketch results of experiments on the THz-transmission and polarized photoluminescence in which the spin orientation by electric current in quantum well 
a)

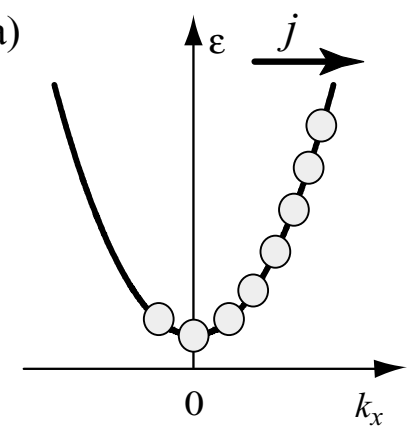

b)

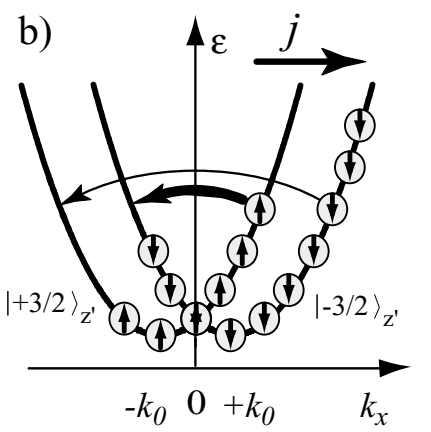

Fig. 9. Comparison of current flow in (a) spin-degenerate and (b) spin-split subbands. (a) Electron distribution at a stationary current flow due to acceleration in an electric field and momentum relaxation. (b) Spin polarization due to spin-flip scattering. Here only $\beta \sigma_{z} k_{x}$ term is taken into account in the Hamiltonian which splits a valence subband into two parabolas with spin-up $|+3 / 2\rangle_{z}$ and spin-down $|-3 / 2\rangle_{z}$ in $z$-direction. Biasing along $x$-direction causes an asymmetric in $\boldsymbol{k}$-space occupation of both parabolas. After Ref. 26.

structures was initially observed.

\subsection{THz-transmission}

In order to observe current induced spin polarization in Refs. 26, 34 linear-circular dichroism and Faraday rotation of terahertz radiation transmitted at normal incidence through samples containing multiple $p$-type QWs were studied (for $n$-type QWs we used mid-infrared radiation). This method allows to detect spin polarization in the growth direction. As material we have chosen GaAs QWs of $\mathrm{C}_{s}$ point group symmetry. This was achieved in $p$-type samples by growing modulation $\mathrm{Si}$ doped QWs on (113)A- or miscut (001)-oriented GaAs substrates. Two kinds of $p$-type samples were investigated. Sample A: (113)A with QWs of width $L_{W}=10$ $\mathrm{nm}$, and sample B: miscut (001) with $L_{W}=20 \mathrm{~nm}$. Samples of $n$-type were prepared on (110)-oriented substrates. They contain asymmetric doped QWs of $8.2 \mathrm{~nm}$ width. To cope with the small Faraday rotation angles of an individual quantum well, we fabricated multiple QW structures containing 100 or 400 QWs. The sample edges were oriented along $[1 \overline{1} 0]$ in the QW plane ( $x$-axis) and perpendicular to this direction ( $y^{\prime}$-axis). Two pairs of ohmic contacts were centered along opposite sample edges of $5 \mathrm{~mm}$ width. In addition structures containing 100 QWs and having very thin barriers were taken as quasi-bulk reference samples.

A spin polarization is not expected for both current directions. For materials of the symmetry used here only an electric current along $x \|$ [11 0$]$-direction is expected to align spins in $z^{\prime}$-direction. By symmetry arguments it is straightforward to show that a current density $j_{x}$ in the plane of the QW yields an average spin polarization $S_{z^{\prime}}$ normal to the QW plane according to $S_{z^{\prime}}=R_{z^{\prime} x} j_{x}$, where $\boldsymbol{R}$ is a second rank pseudo-tensor ${ }^{7}$. However, for a current flowing along $y^{\prime}$-direction, $S_{z^{\prime}}=0$ holds, 

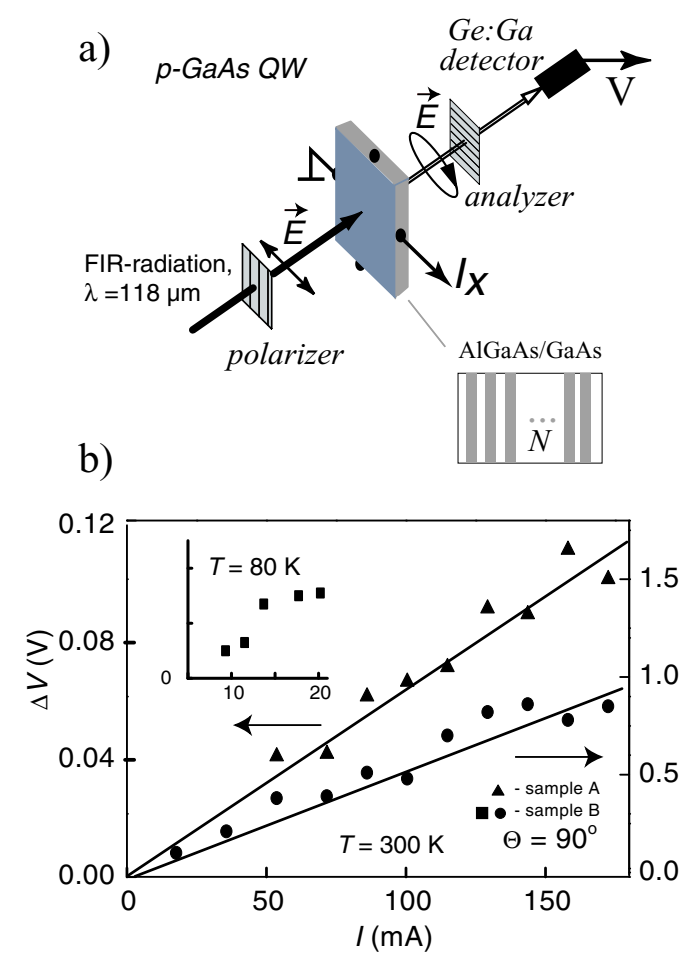

Fig. 10. (a) Experimental set-up. (b) polarization dependent signal for current in active direction as a function of current strength for two samples. Inset shows the same dependence $(\Delta V(I))$ for sample B at $T=80 \mathrm{~K}$. After Ref. 34 .

since due to symmetry $R_{z^{\prime} y^{\prime}}=0$. Thus a spin polarization may occur for current flow in one but not in the other direction. Below we denote these directions as active and passive, respectively.

The transmission measurements were carried out at room temperature and at $T=80 \mathrm{~K}$ using linearly polarized $\lambda=118 \mu \mathrm{m}$ radiation for $p$-type samples and mid-IR light with $\lambda \approx 9 \mu \mathrm{m}$ for $n$-type samples. The electric current was applied as $10 \mu \mathrm{s}$ long pulses with a repetition rate of $20 \mathrm{kHz}$. The schematic experimental set up is shown in Fig. 10(a): the sample was placed between two metallic grid polarizers and the cw-terahertz radiation was passed through this optical arrangement. The transmitted radiation was detected in-phase with the current modulation frequency using a highly sensitive Ge:Ga extrinsic photodetector operated at $4.2 \mathrm{~K}$.

The signal $\Delta V$ caused by rotation of polarization plane was observed only for currents flowing in the active direction. The spin polarization induced signals for samples A and B are shown in Fig. 10(b). At room temperature and current $I=$ $150 \mathrm{~mA}$ we obtain a Faraday rotation angle per quantum well of $0.4 \mathrm{mrad}$ for sample $\mathrm{A}$ and $0.15 \mathrm{mrad}$ for sample B. Lowering the temperature of sample B to $80 \mathrm{~K}$ we 


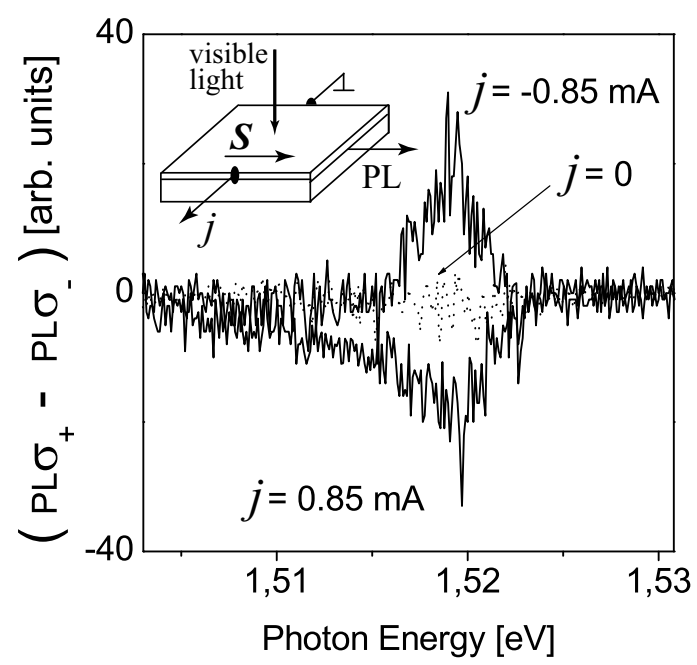

Fig. 11. Differential spectra of polarized PL for two current directions (after Ref. 27). Base line is taken with the current turned off. Inset shows experimental geometry.

obtain two times larger Faraday rotation. While the experiment displays clear spin polarization due to the driving current, there is not yet a straightforward way at hand to extract the value of the spin polarization from the Faraday rotation angle.

According to the theory of Aronov and Lyanda-Geller, ${ }^{24}$ a current should yield a spin polarization of the order of $\langle S\rangle \approx \beta \cdot\langle k\rangle / k_{\mathrm{B}} T$. Using Eq. (15) we estimate this value as:

$$
\langle S\rangle=\frac{Q \beta}{k_{\mathrm{B}} T} \cdot \frac{m^{*}}{e \hbar p} j,
$$

where $Q \simeq 1$ is a constant determined by momentum scattering and the spin relaxation mechanism. ${ }^{31}$ For a situation where Fermi statistic applies the factor $k_{\mathrm{B}} T$ needs to be replaced by $2 E_{\mathrm{F}} / 3$. Calculating $\langle S\rangle$ with the experimental parameters $p=2 \cdot 10^{11} \mathrm{~cm}^{-2}, m^{*}=0.2 m_{0}$ and spin splitting constant $\beta=5 \mathrm{meV} \cdot \mathrm{nm}$ (see Ref. 53), we obtain an average spin of $3.2 \cdot 10^{-4}$ and $0.8 \cdot 10^{-4}$ for the experimentally relevant current densities $3 \mathrm{~mA} / \mathrm{cm}$ and $0.75 \mathrm{~mA} / \mathrm{cm}$ per $\mathrm{QW}$, respectively.

\subsection{Polarized luminescence}

In Refs. 27, 33 to detect the inverse spin-galvanic effect the degree of circular polarization of the 2DHG photoluminescence was measured This experimental procedure has become a proven method for probing spin polarization. ${ }^{50}$ The sample cleaved into bars was studied with the current flowing along the long side cleaved parallel to [110] direction. In later experiments also (113)-grown samples were studied. ${ }^{33}$ The photoluminescence (PL) was excited with $633 \mathrm{~nm}$ line from a helium-neon laser. In (001)-oriented samples the PL was collected from the cleaved (110) facet of the sample. On the other hand, at the (113) heterojunctions, because of $\mathrm{C}_{s}$ symmetry, 
the mean spin density will have a component along the growth direction. Therefore, the circularly polarized PL in this case was detected in the back scattering geometry.

In experiments the influence of glancing light along the surface was prevented by detecting the PL through a narrow aperture on the cleaved facet. The degree of circular polarization $P_{\text {circ }}$ was analyzed with a $\lambda / 4$ plate and a linear polarizer. Figure 11 shows the experimental arrangement for measuring the current induced polarization and differential spectra, $\left(\mathrm{PL} \sigma_{+}-\mathrm{PL} \sigma_{-}\right)$, for the two opposing current directions. The observation of the circularly polarized radiation and, in particularly the reversing of helicity upon the reverse of the current direction, demonstrates current induced spin polarization. The observed degree of polarization in (001)grown samples yields a maximum of $2.5 \%$. In (113)-grown samples even higher polarization of $12 \%$ at $5.1 \mathrm{~K}$ is achieved.

\section{Application of the spin-galvanic effect}

Spin-galvanic effect provides experimental access to spin properties of lowdimensional structures. It can be applied to investigation of spin relaxation times for monopolar spin orientation where only one type of charge carrier is involved in the excitation-relaxation process. This condition is close to that of electrical spin injection in semiconductors. This method is based on the Hanle effect in the spin-galvanic current. ${ }^{4} \mathrm{~A}$ further important application of the spin-galvanic was addressed in Ref. 16. It is demonstrated that angular dependent measurements of spin photocurrents allow us to separate Dresselhaus and Rashba terms. The relative strength of these terms is of importance because it is directly linked to the manipulation of the spin of charge carriers in semiconductors, one of the key problems in the field of spintronics. Spin polarization may be tuned by means of the Rashba spin-orbit coupling in quantum wells. In addition to the Rashba coupling, caused by structural inversion asymmetry, also a Dresselhaus type of coupling, caused by a lack of inversion symmetry in the host material, contributes to spin splitting. In $\mathrm{C}_{2 v}$ symmetry these terms are given by symmetric and antisymmetric tensor components $\beta_{\mathrm{BIA}}=\left(\beta_{x y}+\beta_{y x}\right) / 2$ and $\beta_{\mathrm{SIA}}=\left(\beta_{x y}-\beta_{y x}\right) / 2$ (see section 1.3). As was mentioned above, the Dresselhaus term $\beta_{\mathrm{BIA}}$ and the Rashba term $\beta_{\mathrm{SIA}}$ result from bulk inversion asymmetry (BIA) and from structural inversion asymmetry (SIA), respectively.

Both Rashba and Dresselhaus couplings result in spin splitting of the band and give rise to a variety of spin-dependent phenomena which allow us to evaluate the magnitude of the total spin splitting of electron subbands. However, usually it is impossible to extract the relative strengths of Rashba and Dresselhaus terms in the spin-orbit coupling. In obtaining the Rashba coefficient, the Dresselhaus contribution is normally neglected. At the same time, Dresselhaus and Rashba terms can interfere in such a way that macroscopic effects vanish though the individual terms are large. ${ }^{32,54}$ For example, both terms can cancel each other resulting in a 

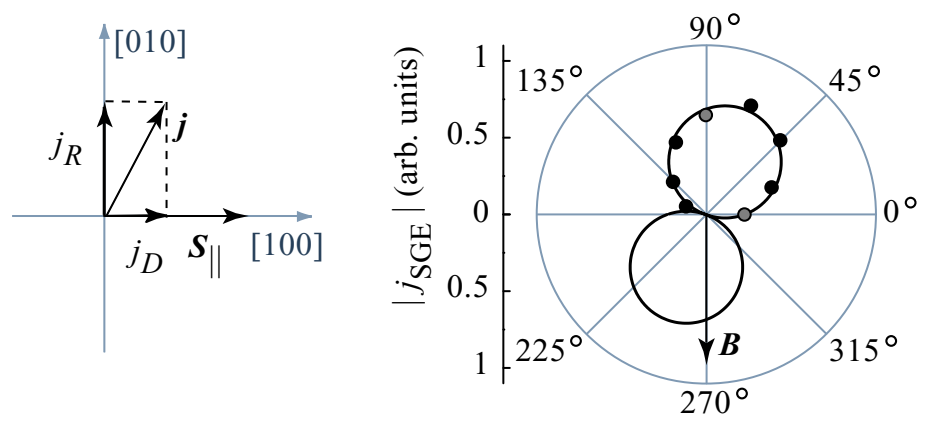

Fig. 12. Photocurrent in $n$-type InAs single QWs at room temperature. The left panel indicates the relation between spin polarization and current contributions for the case of $\boldsymbol{S}_{\|} \|$[100] (after Eq. (18)). The right panel shows measurements of the spin galvanic current as a function of angle $\vartheta$. Data are presented in polar coordinates (after Ref. 16).

vanishing spin splitting in certain $\boldsymbol{k}$-space directions. ${ }^{7}$ This cancellation leads to the disappearance of an antilocalization, ${ }^{55}$ the absence of spin relaxation in specific crystallographic directions, ${ }^{32,56}$ and the lack of Shubnikov-de Haas beating. ${ }^{54}$ In Ref. 57 the importance of both Rashba and Dresselhaus terms was pointed out: tuning $\beta_{\text {SIA }}$ such that $\beta_{\text {SIA }}=\beta_{\text {BIA }}$ holds, allows one to build a nonballistic spin field-effect transistor.

By mapping the magnitude of the spin photocurrent in the plane of a QW the ratio of both terms can directly be determined from experiment and does not relay on theoretically obtained quantities. ${ }^{16}$ Indeed, the spin-galvanic current is driven by the in-plane average spin of electrons $\boldsymbol{S}_{\|}$according to ${ }^{16}$

$$
\boldsymbol{j}_{\mathrm{SGE}} \propto\left(\begin{array}{c}
\beta_{\mathrm{BIA}}-\beta_{\mathrm{SIA}} \\
\beta_{\mathrm{SIA}}-\beta_{\mathrm{BIA}}
\end{array}\right) \boldsymbol{S}_{\|} .
$$

Therefore, the spin-galvanic current $\boldsymbol{j}_{\mathrm{SGE}}$ consists of Rashba and Dresselhaus coupling induced currents, $\boldsymbol{j}_{R}$ and $\boldsymbol{j}_{D}$ (see Fig. 12(a)). Their magnitudes are $j_{R} \propto \beta_{\mathrm{SIA}}\left|\boldsymbol{S}_{\|}\right|, j_{D} \propto \beta_{\mathrm{BIA}}\left|\boldsymbol{S}_{\|}\right|$and their ratio is

$$
j_{R} / j_{D}=\beta_{\mathrm{SIA}} / \beta_{\mathrm{BIA}} .
$$

Figure 12(b) shows the angular dependence of the spin-galvanic current $j_{\mathrm{SGE}}$ measured at room temperature on (001)-oriented $n$-type InAs/ $\mathrm{Al}_{0.3} \mathrm{Ga}_{0.7} \mathrm{Sb}$ single QWs of $15 \mathrm{~nm}$ width. Because of the admixture of helicity independent magnetogyrotropic effects, ${ }^{17,22}$ the spin-galvanic effect is extracted after eliminating current contributions which are helicity independent: $j_{\mathrm{SGE}}=\left(j_{\sigma_{+}}-j_{\sigma_{-}}\right) / 2$.

The nonequilibrium in-plane spin polarization $\boldsymbol{S}_{\|}$is prepared as described in section 1.2: Circularly polarized light at normal incidence on the QW plane polarizes the electrons in the lowest conduction subband resulting in a monopolar spin orientation in the $z$-direction (Fig. 13(b)). An in-plane magnetic field $(B=1 \mathrm{~T})$ rotates 

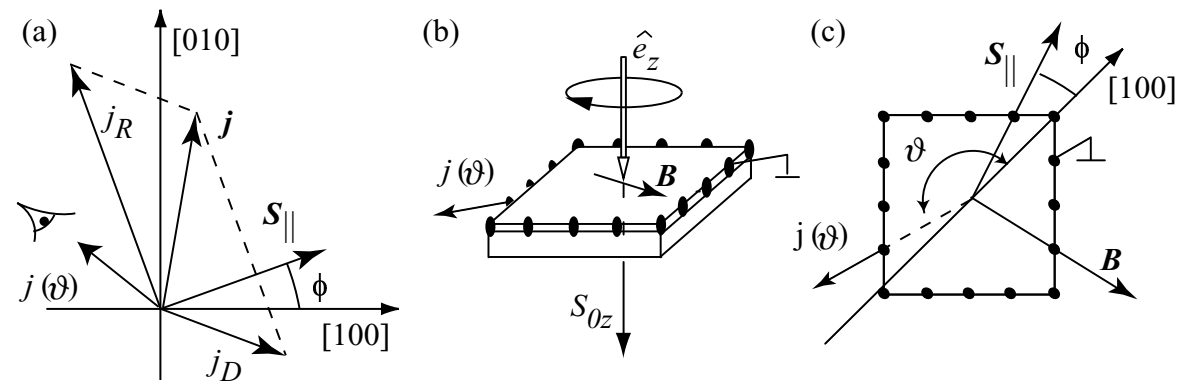

Fig. 13. Angular dependence of the spin-galvanic current (a) and geometry of the experiment (b) and (c). Data are given after Ref. 16.

the spin around the magnetic field axis (precession) and results in a nonequilibrium in-plane spin polarization $S_{\|} \propto \omega_{L} \tau_{s}$. In the range of the applied magnetic field strength the spin-galvanic current rises linearly with $B$ indicating $\omega_{L} \tau_{s}<1$ and, thus, the Hanle denominator in $\boldsymbol{S}$ can be neglected. The angle between the magnetic field and $\boldsymbol{S}_{\|}$in general depends on details of the spin relaxation process. In these InAs QW structures, the isotropic Elliott-Yafet spin relaxation mechanism dominates. ${ }^{32}$ Thus the in-plane spin polarization $\boldsymbol{S}_{\|}$is always perpendicular to $\boldsymbol{B}$ and can be varied by rotating $\boldsymbol{B}$ around $z$ as illustrated in Fig. 13(c).

To obtain the Rashba and Dresselhaus contributions the spin-galvanic effect is measured for a fixed orientation of $\boldsymbol{S}_{\|}$for all accessible directions $\vartheta$ (see Fig. 13(c)). As discussed above the current $\boldsymbol{j}_{R}$ always flows perpendicularly to the spin polarization $\boldsymbol{S}_{\|}$, and $\boldsymbol{j}_{D}$ encloses an angle $-2 \phi$ with $\boldsymbol{S}_{\|}$. Here $\phi$ is the angle between $\boldsymbol{S}_{\|}$ and the $x$-axis. Then, the current component along any direction given by the angle $\vartheta$ can be written as a sum of the projections of $\boldsymbol{j}_{R}$ and $\boldsymbol{j}_{D}$ on this direction

$$
j_{\mathrm{SGE}}(\vartheta)=j_{D} \cos (\vartheta+\phi)+j_{R} \sin (\vartheta-\phi) .
$$

Evaluating measurements using this equation immediately yields the ratio between Rashba and Dresselhaus terms. Three directions of spin population $\boldsymbol{S}_{\|}$are particularly suited to extract the ratio between Rashba and Dresselhaus terms. In the first geometry sketched in the left panel of Fig. 12, the spin polarization $\boldsymbol{S}_{\|}$is set along [100] $(\phi=0)$. Then it follows from Eq. (20) that the currents along the [100]direction $(\vartheta=0)$ and [010]-direction $(\vartheta=\pi / 2)$ are equal to $j_{D}$ and $j_{R}$, respectively, as shown on the left-hand side of Fig. 12.

The ratio of Rashba and Dresselhaus currents can be directly read off from the right-hand side of Fig. $12, j_{R} / j_{D}=j(\pi / 2) / j(0)$. The value obtained by this method in Ref. 16 for InAs QW structure is $j_{R} / j_{D}=\beta_{\text {SIA }} / \beta_{\text {BIA }}=2.1$ in good agreement to theoretical results ${ }^{58}$ which predict a dominating Rashba spin-orbit coupling for InAs QWs and results of $\mathbf{k} \cdot \mathbf{p}$ calculations giving $\beta_{\mathrm{SIA}} / \beta_{\mathrm{BIA}}=1.85$ (see Ref. 59). Applying external gate voltage the $\boldsymbol{j}_{R}$ current contribution can be tuned by the external gate voltage ${ }^{60}$ which is naturally, because Rashba constant 
can be controlled by the electric field normal to the plane of the heterostructure. ${ }^{61}$

In the second procedure a nonequilibrium spin polarization is induced along [110]-axis $(\phi=\pi / 4)$ or [11 0$](\phi=-\pi / 4)$ resulting in the maximum value $j=j_{R}-j_{D}$ or $j_{R}+j_{D}$, respectively. These values also allow a straightforward determination of $j_{R} / j_{D}=\beta_{\mathrm{SIA}} / \beta_{\mathrm{BIA}}$.

\section{Summary}

A nonequilibrium uniform spin polarization drives an electric current and viceversa in QWs if they belong to a gyrotropic crystal class. The current flow in the spin-galvanic effect is driven by asymmetric spin relaxation of a homogeneous nonequilibrium spin polarization. Macroscopic measurements of spin-galvanic effect in different geometric configurations of experiments allow us to conclude on details of the microscopic tensorial spin-orbit interaction. In particular the relation between the Dresselhaus-like terms and the Rashba term may be obtained. Furthermore the macroscopic in-plain symmetry of QWs may easily be determined. Spin-galvanic effect was also applied to investigate the mechanism of monopolar spin orientation where only one type of charge carriers is involved in the excitation-relaxation process. The inverse spin-galvanic effect demonstrates that substantial spin orientation can be obtained in all-electric scheme based on non-magnetic semiconductor structures.

\section{Acknowledgements}

This work was supported by the DFG via Collaborative Research Center SFB689. The author would like to thank W. Prettl, V.V. Bel'kov, E.L. Ivchenko, L.E. Golub, S.A. Tarasenko, S.N. Danilov, S. Giglbereger, W. Wegsheider and D. Weiss for active help and discussions. I also would like to thank A.Yu. Silov for kindly provided experimental plot with experimental data on PL-measurements.

1. Y.A. Bychkov and E.I. Rashba, Pis'ma Zh. Èksp. Teor. Fiz. 39, 66 (1984) [JETP Lett. 39, 78 (1984)].

2. I. Zutic, J. Fabian and S. Das Sarma, Rev. Modern Phys. 76, 323 (2004).

3. E.L. Ivchenko, Yu.B. Lyanda-Geller and G.E. Pikus, Pis'ma Zh. Èksp. Teor. Fiz. 50, 156 (1989) [JETP Lett. 50, 175 (1989)].

4. S.D. Ganichev, E.L. Ivchenko, V.V. Bel'kov, S.A. Tarasenko, M. Sollinger, D. Weiss, W. Wegscheider and W. Prettl, Nature (London) 417, 153 (2002).

5. S.D. Ganichev and W. Prettl, Intense Terahertz Excitation of Semiconductors (Oxford University Press, Oxford, 2006).

6. E.L. Ivchenko, Optical Spectroscopy of Semiconductor Nanostructures (Alpha Science Int., Harrow, UK, 2005).

7. S.D. Ganichev and W. Prettl, J. Phys.: Condens. Matter 15, R935 (2003).

8. S.D. Ganichev, E.L. Ivchenko, S.N. Danilov, J. Eroms, W. Wegscheider, D. Weiss and W. Prettl, Phys. Rev. Lett. 86, 4358 (2001).

9. S.D. Ganichev, S.N. Danilov, V.V. Bel'kov, E.L. Ivchenko, M. Bichler, W. Wegscheider, D. Weiss and W. Prettl, Phys. Rev. Lett. 88, 057401 (2002). 
10. V.V. Bel'kov, S.D. Ganichev, Petra Schneider, C. Back, M. Oestreich, J. Rudolph, D. Hägele, L.E. Golub, W. Wegscheider and W. Prettl, Solid State Commun. 128, 283 (2003).

11. M. Sasaki, Y. Ohno and H. Ohno, in Proc. 9th Symp. on the Physics and application of spin-related phenomena in semiconductors, PASPS-9 (Tokyo, Japan 2003), p. 176.

12. S.D. Ganichev, Petra Schneider, V.V. Bel'kov, E.L. Ivchenko, S.A. Tarasenko, W. Wegscheider, D. Weiss, D. Schuh, D.G. Clarke, M. Merrick, B.N. Murdin, P. Murzyn, P.J. Phillips, C.R. Pidgeon, E.V. Beregulin and W. Prettl, Phys. Rev. B 68, R081302 (2003).

13. J. Hübner, W.W. Rühle, M. Klude, D. Hommel, R.D.R. Bhat, J.E. Sipe and H.M. van Driel, Phys. Rev. Lett. 90, 216601 (2003).

14. M.J. Stevens, A.L. Smirl, R.D.R. Bhat, A. Najimaie, J.E. Sipe and H.M. van Driel, Phys. Rev. Lett. 90, 136603 (2003).

15. L.E. Golub, Phys. Rev. B 67, 235320 (2003).

16. S.D. Ganichev, V.V. Bel'kov, L.E. Golub, E.L. Ivchenko, Petra Schneider, S. Giglberger, J. Eroms, J. De Boeck, G. Borghs, W. Wegscheider, D. Weiss and W. Prettl, Phys. Rev. Lett. 92, 256601 (2004).

17. V.V. Bel'kov, S.D. Ganichev, E.L. Ivchenko, S.A. Tarasenko, W. Weber, S. Giglberger, M. Olteanu, P. Tranitz, S.N. Danilov, Petra Schneider, W. Wegscheider, D. Weiss and W. Prettl, J. Phys.: Condens. Matter, 17, 3405 (2005).

18. M. Bieler, N. Laman, H.M. van Driel and A.L. Smirl, Appl. Phys. Lett. 86, 061102 (2005)).

19. S.A. Tarasenko and E.L. Ivchenko, Pis'ma Zh. Èksp. Teor. Fiz. 81, 292 (2005) [JETP Lett. 81, $231(2005)]$.

20. W. Weber, S.D. Ganichev, Z.D. Kvon, V.V. Bel'kov, L.E. Golub, S.N. Danilov, D. Weiss, W. Prettl, Hyun-Ick Cho and Jung-Hee Lee, Appl. Phys. Lett. 87, 262106 (2005)

21. C.L. Yang, H.T. He, Lu Ding, L.J. Cui, Y.P. Zeng, J.N. Wang and W.K. Ge, Phys. Rev. Lett. 96, 186605 (2006).

22. S.D. Ganichev, V.V. Bel'kov, S.A. Tarasenko, S.N. Danilov, S. Giglberger, Ch. Hoffmann, E.L. Ivchenko, D. Weiss, W. Wegscheider, Ch. Gerl, D. Schuh, J. Stahl, J.De Boeck, G. Borghs and W. Prettl, "Zero-bias spin separation", Nature Phys. 2, 609 (2006).

23. S.D. Ganichev, V.V. Bel'kov, Petra Schneider, E.L. Ivchenko, S.A. Tarasenko, D. Schuh, W. Wegscheider, D. Weiss and W. Prettl, Phys. Rev. B 68, 035319 (2003).

24. A.G. Aronov and Yu.B. Lyanda-Geller, Pis'ma Zh. Èksp. Teor. Fiz. 50, 398 (1989) [JETP Lett. 50, 431 (1989)].

25. V.M. Edelstein, Solid State Commun. 73, 233 (1990).

26. S.D. Ganichev, S.N. Danilov, Petra Schneider, V.V. Bel'kov, L.E. Golub, W. Wegscheider, D. Weiss and W. Prettl, cond-mat/0403641 (2004).

27. A.Yu. Silov, P.A. Blajnov, J.H. Wolter, R. Hey, K.H. Ploog and N.S. Averkiev, Appl. Phys. Lett. 85, 5929 (2004).

28. Y.K. Kato, R.C. Myers, A.C. Gossard and D.D. Awschalom, Phys. Rev. Lett. 93, $176601(2004)$

29. L.E. Vorob'ev, E.L. Ivchenko, G.E. Pikus, I.I. Farbstein, V.A. Shalygin and A.V. Sturbin, Pisma Zh. Eksp. Teor. Fiz. 29, 485 (1979) [JETP Lett. 29, 441 (1979)].

30. E.L. Ivchenko and G.E. Pikus, Pisma Zh. Eksp. Teor. Fiz. 27, 640 (1978) [JETP Lett. 27, 604 (1978)].

31. A.G. Aronov, Yu.B. Lyanda-Geller and G.E. Pikus, Zh. Eksp. Teor. Fiz. 100, 973 (1991) [Sov. Phys. JETP 73, 537 (1991)]. 
32. N.S. Averkiev, L.E. Golub and M. Willander, J. Phys.: Condens. Matter 14, R271 (2002).

33. A.Yu. Silov, P.A. Blajnov, J.H. Wolter, R. Hey, K.H. Ploog and N.S. Averkiev, in Proc. 13th Int. Symp. Nanostructures: Phys. and Technol., (St. Petersburg, Russia, 2005).

34. S.D. Ganichev, S.N. Danilov, Petra Schneider, V.V. Bel'kov, L.E. Golub, W. Wegscheider, D. Weiss and W. Prettl, J. Magn. and Magn. Materials 300, 127 (2006).

35. N.P. Stern, S. Ghash, G. Xiang, M. Zhu and D.D. Awschalom, cond-mat/0607288 (2006).

36. M.I. D'yakonov and V.Yu. Kachorovskii, Fiz. Tekh. Poluprovodn. 20, 178 (1986) [Sov. Phys. Semicond. 20, 110 (1986)].

37. O. Krebs and P. Voisin, Phys. Rev. Lett. 77, 1829 (1996).

38. F. Meier and B.P. Zakharchenya, eds., Optical Orientation, in series Modern Problems in Condensed Matter Sciences, Vol. 8 ed. by V.M. Agranovich and A.A. Maradudin, (Elsevier Science Publ., Amsterdam, 1984).

39. W. Hanle, Z. Physik 30, 93 (1924).

40. S.D. Ganichev, E.L. Ivchenko, V.V. Bel'kov, S.A. Tarasenko, M. Sollinger, D. Schowalter, D. Weiss, W. Wegscheider and W. Prettl, J. of Supercond.: Incorporating Novel Magn. 16, 369 (2003).

41. X. Marie, T. Amand, P. Le Jeune, M. Paillard, P. Renucci, L.E. Golub, V.D. Dymnikov and E.L. Ivchenko, Phys. Rev. B 60, 5811 (1999).

42. A.V. Andrianov and I.D. Yaroshetskii, Pis'ma Zh. Èksp. Teor. Fiz. 40, 131 (1984) [Sov. Phys. JETP 40, 882 (1984)].

43. E.L. Ivchenko, Yu.B. Lyanda-Geller and G.E. Pikus, Solid State Commun. 69, 663 (1989).

44. S.A. Tarasenko, E.L. Ivchenko, V.V. Bel'kov, S.D. Ganichev, D. Schowalter, Petra Schneider, M. Sollinger, W. Prettl, V.M. Ustinov, A.E. Zhukov and L.E. Vorobjev, cond-mat/0301393 (2003).

45. S.A. Tarasenko, E.L. Ivchenko, V.V. Bel'kov, S.D. Ganichev, D. Schowalter, Petra Schneider, M. Sollinger, W. Prettl, V.M. Ustinov, A.E. Zhukov and L.E. Vorobjev, J. of Supercond.: Incorporating Novel Magn. 16, 419 (2003).

46. E.L. Ivchenko and S.A. Tarasenko, Zh. Ėksp. Teor. Fiz. 126, 426 (2004) [JETP 99, 379 (2004)].

47. E.L. Ivchenko and G.E. Pikus, Superlattices and Other Heterostructures. Symmetry and Optical Phenomena (Springer, Berlin, 1997).

48. G.M.H. Knippels, X. Yan, A.M. MacLeod, W.A. Gillespie, M. Yasumoto, D. Oepts and A.F.G. van der Meer, Phys. Rev. Lett. 83, 1578 (1999).

49. R.R. Parson, Can. J. Phys. 49, 1850 (1971).

50. Semiconductor Spintronics and Quantum Computation, eds. D.D. Awschalom, D. Loss and N. Samarth, in the series Nanoscience and technology, eds. K. von Klitzing, H. Sakaki and R. Wiesendanger (Springer, Berlin, 2002).

51. R. Winkler, Spin-Orbit Coupling Effects in Two-Dimensional Electron and Hole Systems, in Springer Tracts in Modern Physics Vol.191 (Springer, Berlin, 2003).

52. W. Zawadzki and P. Pfeffer, Semicond. Sci. Technol. 19, R1 (2004).

53. P. Schneider, J. Kainz, S.D. Ganichev, V.V. Bel'kov, S.N. Danilov, M.M. Glazov, L.E. Golub, U. Rössler, W. Wegscheider, D. Weiss, D. Schuh and W. Prettl, J. Appl. Phys. 96, 420 (2004).

54. S.A. Tarasenko and N.S. Averkiev, Pis'ma Zh. Èksp. Teor. Fiz. 75, 669 (2002) [JETP Lett. 75, 552 (2002)].

55. W. Knap, C. Skierbiszewski, A. Zduniak, E. Litwin-Staszewska, D. Bertho, F. Kobbi, 
J.L. Robert, G.E. Pikus, F.G. Pikus, S.V. Iordanskii, V. Mosser, K. Zekentes and Yu.B. Lyanda-Geller, Phys. Rev. B 53, 3912 (1996).

56. N.S. Averkiev and L.E. Golub, Phys. Rev. B 60, 15582 (1999).

57. J. Schliemann, J.C. Egues and D. Loss, Phys. Rev. Lett. 90, 146801 (2003).

58. G. Lommer, F. Malcher and U. Rössler, Phys. Rev. Lett. 60, 728 (1988).

59. P. Pfeffer and W. Zawadzki, Phys. Rev. B 59, R5312 (1999).

60. S. Giglberger, S.D. Ganichev, V.V. Bel'kov, M. Koch, T. Kleine-Ostmann, K. Pierz, E.L. Ivchenko, L.E. Golub, S.A. Tarasenko and W. Prettl, Proc. 13th Int. Symp. Nanostructures: Physics and Technology, (St. Petersburg, Russia, 2005), p. 65.

61. J. Nitta, T. Akazaki, H. Takayanagi and T. Enoki, Phys. Rev. Lett. 78, 1335 (1997). 